\title{
Approximate Complete Solutions of DKP Equation under a Vector Exponential Interaction via a Pekeris-Type Approximation
}

\author{
S. Zarrinkamar, A. A. Rajabi, and H. Hassanabadi \\ Physics Department, Shahrood University of Technology, P.O. Box 3619995161-316, \\ Shahrood 3619995161, Iran \\ Correspondence should be addressed to S. Zarrinkamar, zarrinkamar.s@gmail.com \\ Received 19 September 2012; Accepted 9 October 2012 \\ Academic Editors: C. Ahn and W. Li \\ Copyright (C) 2012 S. Zarrinkamar et al. This is an open access article distributed under the Creative \\ Commons Attribution License, which permits unrestricted use, distribution, and reproduction in \\ any medium, provided the original work is properly cited. \\ The DKP equation for an exponential potential is exactly solved via an appropriate approximation \\ using the methodology of supersymmetric quantum mechanics. We see that the solutions are \\ already known without any cumbersome algebra we face in any numerical or analytical approach. \\ Closed forms of eigenfunctions and eigenvalues are reported.
}

\section{Introduction}

Working on the basis of Dirac and Klein-Gordon equations, spin- 0 and spin- $1 / 2$ particles have been extensively discussed via many analytical and numerical techniques. For the spin1 particles, however, there are only few investigations. The main reason for this lack of literature is probably the mathematical complexity of Proca equation describing the spin1 particles. The Duffin-Kemmer-Petiau (DKP) [1-4] equation, however, provides us with a theoretical basis for describing both spin-0 and spin-1 particles on a relatively easier background. For many years, the DKP equation was thought to be exactly equivalent to KleinGordon (KG) and Proca equations and consequently was in the shadow of them. Now, we know that the equations are not exactly the same and the equivalence does not hold generally [5-14]. In addition, the DKP equation is richer in the investigation of interactions and is even closer to some experimental data in comparison with KG or Proca equations [15-20]. Moreover, besides cosmology and gravity, this equation has been tested in many branches of physics including particle and nuclear physics [21-25].

As usual, the most appealing case studies are Coulomb and quadratic terms [2628], and other ones including the woods-Saxon and Hulthen are investigated by different 
approaches as well [29-32]. Within the present work, we first review the DKP equation. Next, an introductory section is included on supersymmetry (SUSY) quantum mechanics. In the last part, we obtain the approximate analytical solutions of the problem.

\section{DKP Equation}

For the sake of briefness, our starting square is

$$
\left(\beta \cdot \vec{p} c+m c^{2}+U_{s}+\beta^{0} U_{v}^{o}\right) \psi(\vec{r})=\beta^{0} E \psi(\vec{r})
$$

where

$$
\psi(\vec{r})=\left(\begin{array}{c}
\psi_{\text {upper }} \\
i \psi_{\text {lower }}
\end{array}\right),
$$

with the upper and lower components, respectively, being

$$
\begin{gathered}
\psi_{\text {upper }} \equiv\left(\begin{array}{l}
\phi \\
\varphi
\end{array}\right), \\
\psi_{\text {lower }} \equiv\left(\begin{array}{l}
A_{1} \\
A_{2} \\
A_{3}
\end{array}\right),
\end{gathered}
$$

$\beta^{0}$ is the usual $5 \times 5$ matrix, and $U_{s}, U_{v}^{o}$ denote the scalar and vector interactions. The equation, in $(3+0)$-dimensions, is therefore written as

$$
\begin{gathered}
\left(m c^{2}+U_{s}\right) \phi=\left(E-U_{v}^{o}\right) \varphi+\hbar c \vec{\nabla} \cdot \vec{A}, \\
\vec{\nabla} \phi=\left(m c^{2}+U_{s}\right) \vec{A}, \\
\left(m c^{2}+U_{s}\right) \varphi=\left(E-U_{v}^{o}\right) \phi,
\end{gathered}
$$

where $\vec{A}=\left(A_{1}, A_{2}, A_{3}\right)$. It should be noted that $\psi$ is a simultaneous eigenfunction of $J^{2}$ and $J_{3}$, that is

$$
\begin{gathered}
J^{2}\left(\begin{array}{l}
\psi_{\text {upper }} \\
\psi_{\text {lower }}
\end{array}\right)=\left(\begin{array}{c}
L^{2} \psi_{\text {upper }} \\
(L+S)^{2} \psi_{\text {lower }}
\end{array}\right)=J(J+1)\left(\begin{array}{l}
\psi_{\text {upper }} \\
\psi_{\text {lower }}
\end{array}\right), \\
J_{3}\left(\begin{array}{c}
\psi_{\text {upper }} \\
\psi_{\text {lower }}
\end{array}\right)=\left(\begin{array}{c}
L_{3} \psi_{\text {upper }} \\
\left(L_{3}+s_{3}\right) \psi_{\text {lower }}
\end{array}\right)=M\left(\begin{array}{l}
\psi_{\text {upper }} \\
\psi_{\text {lower }}
\end{array}\right),
\end{gathered}
$$


and the general solution is

$$
\psi_{J M}(r)=\left(\begin{array}{c}
f_{n J}(r) Y_{J M}(\Omega) \\
g_{n J}(r) Y_{J M}(\Omega) \\
i \sum_{L} h_{n J L}(r) Y_{J L 1}^{M}(\Omega)
\end{array}\right)
$$

where the spherical harmonics $Y_{J M}(\Omega)$ are of order $J, Y_{J L 1}^{M}(\Omega)$ are the normalized vector spherical harmonics, and $f_{n J}, g_{n J}$, and $h_{n J L}$ denote the radial wave functions. It is shown that the above equations result in the coupled differential equations [26, 33]:

$$
\begin{gathered}
\left(E-U_{v}^{0}\right) F(r)=\left(m c^{2}+U_{s}\right) G(r), \\
\left(\frac{d F(r)}{d r}-\frac{J+1}{r} F(r)\right)=-\frac{1}{\alpha_{J}}\left(m c^{2}+U_{s}\right) H_{1}(r), \\
\left(\frac{d F(r)}{d r}+\frac{J}{r} F(r)\right)=\frac{1}{\zeta}\left(m c^{2}+U_{s}\right) H_{-1}(r), \\
-\alpha_{J}\left(\frac{d H_{1}(r)}{d r}+\frac{J+1}{r} H_{1}(r)\right)+\zeta\left(\frac{d H_{-1}(r)}{d r}-\frac{J}{r} H_{-1}(r)\right) \\
=\frac{1}{\hbar c}\left(\left(m c^{2}+U_{s}\right) F(r)-\left(E-U_{v}^{0}\right) G(r)\right),
\end{gathered}
$$

which give

$$
\begin{aligned}
& \frac{d^{2} F(r)}{d r^{2}}\left[1+\frac{\zeta_{J}^{2}}{\alpha_{J}^{2}}\right]-\frac{d F(r)}{d r}\left[\frac{U_{s}^{\prime}}{\left(m+U_{s}\right)}\left(1+\frac{\zeta_{J}^{2}}{\alpha_{J}^{2}}\right)\right] \\
& +F(r)\left[-\frac{J(J+1)}{r^{2}}\left(1+\frac{\zeta_{J}^{2}}{\alpha_{J}^{2}}\right)+\frac{U_{s}^{\prime}}{\left(m+U_{s}\right)}\left(\frac{J+1}{r}-\frac{\zeta_{J}^{2}}{\alpha_{J}^{2}} \frac{J}{r}\right)\right] \\
& -\left[\frac{1}{\alpha_{J}^{2}}\left(\left(m+U_{s}\right)^{2}-\left(E-U_{v}^{0}\right)^{2}\right)\right]=0,
\end{aligned}
$$

where $\alpha_{J}=\sqrt{(J+1) /(2 J+1)}$ and $\zeta_{J}=\sqrt{J /(2 J+1)}$. In the case of $U_{s}=0$, we recover the well-known formula

$$
\left(\frac{d^{2}}{d r^{2}}-\frac{J(J+1)}{r^{2}}+\left(E-U_{v}^{0}\right)^{2}-m^{2}\right) F(r)=0 .
$$

Within the next section, we give a brief introduction to the SUSY method. 


\subsection{SUSY and Shape Invariance}

The basic idea of the SUSY approach is based on finding the solutions of the Riccati equation

$$
V_{\mp}=\Phi^{2} \mp \Phi^{\prime},
$$

with $V$ being the potential of Schrödinger equation. If the condition

$$
V_{+}\left(a_{0}, x\right)=V_{-}\left(a_{1}, x\right)+R\left(a_{1}\right)
$$

is satisfied, we call the partner Hamiltonians shape invariant. In the latter relation, $a_{1}$ is a new set of parameters uniquely determined from the old set $a_{0}$ via the mapping $F: a_{0} \mapsto a_{1}=$ $F\left(a_{0}\right)$ and the residual term $R\left(a_{1}\right)$ does not include $x$. Actually, the shape invariance implies that the partner potential, apart from some constant terms, is interpreted as a new partner potential $V_{-}\left(a_{1}, x\right)$ associated with a new SUSY potential $\Phi\left(a_{1}, x\right)$ [34]. In such a case, the problem is simplified to a high degree and everything of interest is calculated via the elegant idea of [34-36]:

$$
\begin{gathered}
E_{n}=\sum_{s=1}^{n} R\left(a_{s}\right), \\
\phi_{n}^{-}\left(a_{0}, x\right)=\prod_{s=0}^{n-1}\left(\frac{A^{\dagger}\left(a_{s}\right)}{\left[E_{n}-E_{s}\right]^{1 / 2}}\right) \phi_{0}^{-}\left(a_{n}, x\right), \\
\phi_{0}^{-}\left(a_{n}, x\right)=C \exp \left\{-\int_{0}^{x} d z \Phi\left(a_{n}, z\right)\right\} .
\end{gathered}
$$

That is, the energy eigenvalues as well as the corresponding eigenfunctions are obtained without cumbersome algebra, the

$$
A_{s}^{\dagger}=-\frac{\partial}{\partial x}+\Phi\left(a_{s}, x\right) .
$$

Hence, shape invariance gives the complete and exact information about the spectrum of the bound states of the following Hamiltonians:

$$
H_{s}=-\frac{\partial^{2}}{\partial x^{2}}+V_{-}\left(a_{s}, x\right)+E_{s} .
$$

It should be noted that the energy eigenfunctions

$$
H_{s} \phi_{n-s}^{-}\left(a_{s}, x\right)=E_{n} \phi_{n-s}^{-}\left(a_{s}, x\right), \quad n \geq s,
$$


of this family of Hamiltonians are related by $[3,4]$

$$
\phi_{n-s}^{-}\left(a_{s}, x\right)=\frac{A^{\dagger}}{\left[E_{n}-E_{s}\right]^{1 / 2}} \phi_{n-(s+1)}^{-}\left(a_{s+1}, x\right) .
$$

In other words, everything is obtained without any cumbersome algebra provided that the superpotential is simply found and the shape invariance exists.

\subsection{A Famous SUSY Example}

Within this section, we review an SUSY example. The results can be found in [34-36]. Choosing the superpotential as

$$
\Phi(x)=A-B \exp (-\beta x)
$$

we find

$$
\begin{aligned}
& V_{-}(x)=\Phi^{2}(x)+\Phi^{\prime}(x)=A^{2}+B^{2} \exp (-2 \beta x)-2 B\left(A+\frac{\beta}{2}\right) \exp (-\beta x) \\
& V_{+}(x)=\Phi^{2}(x)-\Phi^{\prime}(x)=A^{2}+B^{2} \exp (-2 \beta x)-2 B\left(A-\frac{\beta}{2}\right) \exp (-\beta x) .
\end{aligned}
$$

Obviously, the partner potentials are shape invariant via a mapping of the form

$$
A_{1}=F(A)=A-\beta
$$

For the energy relation, as

$$
\begin{aligned}
A_{1} & =F(A)=A-\beta, \\
A_{2} & =F\left(A_{1}\right)=A_{1}-\beta=A-2 \beta, \\
\vdots & \\
A_{s} & =F\left(A_{s-1}\right)=A-n \beta,
\end{aligned}
$$

using (2.11) and (2.12), we obtain

$$
\begin{aligned}
E_{n} & =\sum_{s=1}^{n} R\left(A_{s}\right)=R\left(A_{1}\right)+R\left(A_{2}\right)+\cdots+R\left(A_{n}\right) \\
& =\left(A^{2}-A_{1}^{2}\right)+\left(A_{1}^{2}-A_{2}^{2}\right)+\cdots+\left(A_{n-1}^{2}-A_{n}^{2}\right) \\
& =A^{2}-A_{n}^{2}=A^{2}-(A-n \beta)^{2} .
\end{aligned}
$$


For the wave functions, based on (2.13) to (2.18), we have

$$
y^{s-n} \exp \left(\frac{-y}{2}\right) L_{n}^{2 s-2 n}(y)
$$

where

$$
\begin{gathered}
y=\left(\frac{2 B}{\beta}\right) \exp (-\beta x), \\
s=\frac{A}{\beta} .
\end{gathered}
$$

\section{Approximate Analytical Solution of the Radial Part}

We now see that the problem appears as the latter SUSY problem. Choosing the potential as $U_{v}=V e^{-a\left(r-r_{0}\right)}$, we get

$$
\left(\frac{d^{2}}{d r^{2}}-\frac{J(J+1)}{r^{2}}+E^{2}+2 E V \exp \left(-a\left(r-r_{0}\right)\right)+V^{2} \exp \left(-2 a\left(r-r_{0}\right)\right)-m^{2}\right) F(r)=0 .
$$

We wish to emphasize that, on the one hand, exponential-type potentials find application in a wide class of physical sciences including cosmology [37-40], nuclear and particle physics [41-45], solid state [46-48], atomic and molecular physics [49-58], and chemical physics [59, $60]$. On the other hand, the DKP equation itself, as an outstanding relativistic wave equation which well explains both microscale phenomena in particle physics and large-scale ones in cosmology, motivated us to do the present calculations. Naturally, depending on the system under consideration, the phenomenological fits are quite different. To be able to analytically solve the problem, let us use the well-known approximation [61]

$$
\frac{1}{r^{2}} \approx\left(C_{0}+C_{1} e^{-\alpha x}+C_{2} e^{-2 \alpha x}\right)
$$

where

$$
\begin{gathered}
x=\frac{r-r_{0}}{r_{0}} \\
\alpha=a r_{0} \\
C_{0}=\frac{1}{r_{0}^{2}}\left(1-\frac{3}{\alpha}+\frac{3}{\alpha^{2}}\right), \\
C_{1}=\frac{1}{1 r_{0}^{2}}\left(\frac{4}{\alpha}-\frac{6}{\alpha^{2}}\right), \\
C_{2}=\frac{1}{r_{0}^{2}}\left(\frac{-1}{\alpha}+\frac{3}{\alpha^{2}}\right) .
\end{gathered}
$$


We find

$$
\begin{aligned}
& \left(\frac{d^{2}}{d x^{2}}-e^{-\alpha x}\left(C_{1} J(J+1)-2 E V\right) r_{0}^{2}+e^{-2 \alpha x}\left(V^{2}-C_{2} J(J+1)\right) r_{0}^{2}+\left(C_{0} J(J+1)+E^{2}-m^{2}\right) r_{0}^{2}\right) \\
& \quad \times F(x)=0,
\end{aligned}
$$

which is a Schrödinger-like equation corresponding to the Morse potential. Before proceeding further, it should be noted that the choice of the system under consideration definitely puts limitations on the approximation (3.2) via (3.3a), (3.3b) and (3.3c) to yield acceptable results. Here, however, as we intend to give a general background for related studies, we avoid focusing on a special system since the numerical data are quite different from one system, for example, a diatomic molecule, to another such as cosmological model.

Also, there exist many other papers which use Pekeris-type approximations for Schrödinger, Dirac, and KG equations for a lengthy list of potentials including the Hulthen, Eckart, Rosen-Morse, and Pöschl-Teller (see [62-69] and references therein).

In addition, the interested reader might find attractive discussion on the SUSY structure of DKP equation in [70]. Moreover, we wish to address the interesting papers [7176] which first discussed investigation of half-line problems on the basis of full-line SUSY examples. Let us now return to our problem. Comparison of (3.4) with (2.20) indicates the correspondence

$$
\begin{gathered}
B= \pm r_{0} \sqrt{\left(V^{2}-C_{2} J(J+1)\right)}, \\
A= \pm r_{0} \sqrt{C_{0} J(J+1)}, \\
\beta=\frac{r_{0}^{2}\left(C_{1} J(J+1)-2 E V\right)}{B}-2 A .
\end{gathered}
$$

Therefore, the eigenfunctions are

$$
\begin{gathered}
F(x)=C_{n} y^{s-n} \exp \left(\frac{-y}{2}\right) L_{n}^{2 s-2 n}(y), \\
y=\left(\frac{2 B}{\beta}\right) \exp (-\beta x), \\
s=\frac{A}{\beta},
\end{gathered}
$$

and the energy eigenvalues satisfy

$$
\begin{aligned}
\varepsilon_{n} & =r_{0}^{2}\left(E^{2}-m^{2}\right)-\lambda_{0}=\cdots \\
& =C_{0} J(J+1) r_{0}^{2}-r_{0}\left( \pm \sqrt{C_{0} J(J+1)}-n\left(\frac{\left(C_{1} J(J+1)-2 E V\right)}{ \pm \sqrt{\left(V^{2}-C_{2} J(J+1)\right)}} \mp 2 \sqrt{C_{0} J(J+1)}\right)\right)^{2} .
\end{aligned}
$$




\section{Conclusion}

We obtained approximate analytical solutions of the DKP equation for an exponential term which in many cases provides more exact solutions than linear or quadratic terms. The results are applicable to many branches of physics from cosmology to particle physics.

\section{References}

[1] N. Kemmer, "Quantum theory of Einstein-Bose particles and nuclear interaction," Proceedings of the Royal Society A, vol. 166, no. 924, pp. 127-153, 1938.

[2] R. J. Duffin, "On the characteristic matrices of covariant systems," Physical Review, vol. 54, no. 12, p. $1114,1938$.

[3] N. Kemmer, "The particle aspect of meson theory," Proceedings of the Royal Society A, vol. 173, no. 952, pp. 91-116, 1939.

[4] G. Petiau, "University of Paris thesis," Académie Royale De Belgique. Classe Des Sciences. Mémoires. Collection, vol. 16, p. 1114, 1936.

[5] T. R. Cardoso, L. B. Castro, and A. S. de Castro, "Effects due to a scalar coupling on the particle-antiparticle production in the Duffin-Kemmer-Petiau theory," International Journal of Theoretical Physics, vol. 49, no. 1, pp. 10-17, 2010.

[6] T. R. Cardoso and A. S. de Castro, "On the threshold for the pair production and localization of spinless particles," Revista Brasileira De Ensino De Fi'Sica, vol. 29, pp. 203-208, 2007.

[7] T. R. Cardoso, L. B. Castro, and A. S. de Castro, "Inconsistencies of a purported probability current in the Duffin-Kemmer-Petiau theory," Physics Letters A, vol. 372, no. 38, pp. 5964-5967, 2008.

[8] L. Chetouani, M. Merad, T. Boudjedaa, and A. Lecheheb, "Solution of Duffin-Kemmer-Petiau equation for the step potential," International Journal of Theoretical Physics, vol. 43, no. 4, pp. 1147-1159, 2004.

[9] T. R. Cardoso, L. B. Castro, and A. S. de Castro, "On the nonminimal vector coupling in the DuffinKemmer-Petiau theory and the confinement of massive bosons by a linear potential," Journal of Physics A, vol. 43, no. 5, Article ID 055306, 2010.

[10] A. S. de Castro, "Bound states of the Duffin-Kemmer-Petiau equation with a mixed minimal-nonminimal vector cusp potential," Journal of Physics A, vol. 44, no. 3, Article ID 035201, 2011.

[11] M. Nowakowski, "The electromagnetic coupling in Kemmer-Duffin-Petiau theory," Physics Letters A, vol. 244, no. 5, pp. 329-337, 1998.

[12] J. T. Lunardi, B. M. Pimentel, R. G. Teixeira, and J. S. Valverde, "Remarks on Duffin-Kemmer-Petiau theory and gauge invariance," Physics Letters A, vol. 268, no. 3, pp. 165-173, 2000.

[13] M. Riedel, Relativistische Gleichungen Fuer Spin-1-Teilchen, Diplomarbeit, Institute for Theoretical Physics, Johann Wolfgang Goethe-University, Frankfurt, Germany, 1979.

[14] E. Fischbach, M. M. Nieto, and C. K. Scott, "Duffin-Kemmer-Petiau subalgebras: representations and applications," Journal of Mathematical Physics, vol. 14, pp. 1760-1774, 1973.

[15] B. C. Clark et al., "Relativistic impulse approximation for Meson-Nucleus scattering in the KemmerDuffin-Petiau formalism," Physical Review Letters, vol. 55, pp. 592-595, 1985.

[16] G. Kalbermann, "Kemmer-Duffin-Petiau equation approach to pionic atoms," Physical Review C, vol. 34, pp. 2240-2243, 1986.

[17] R. E. Kozack, B. C. Clark, S. Hama et al., "Relativistic deuteron-nucleus scattering in the KemmerDuffin-Petiau formalism," Physical Review C, vol. 37, pp. 2898-2901, 1988.

[18] R. E. Kozack, B. C. Clark, S. Hama et al., "Spin-one Kemmer-Duffin-Petiau equations and intermediate-energy deuteron-nucleus scattering," Physical Review C, vol. 40, pp. 2181-2194, 1989.

[19] V. K. Mishra, B. C. Clark, S. Hama et al., "Implications of various spin-one relativistic wave equations for intermediate-energy deuteron-nucleus scattering," Physical Review C, vol. 43, pp. 801-811, 1991.

[20] B. C. Clarka, R. J. Furnstahla, L. K. Kerr et al., "Pion-nucleus scattering at medium energies with densities from chiral effective field theories," Physics Letters B, vol. 427, no. 3-4, pp. 231-234, 1998.

[21] V. Gribov, "QCD at large and short distance," The European Physical Journal C, vol. 10, pp. 71-90, 1999.

[22] I. V. Kanatchikov, "On the Duffin-Kemmer-Petiau formulation of the covariant Hamiltonian dynamics in field theory," Reports on Mathematical Physics, vol. 46, no. 1-2, pp. 107-112, 2000. 
[23] J. T. Lunardi, B. M. Pimentel, R. G. Teixeira, and J. S. Valverde, "Remarks on Duffin-Kemmer-Petiau theory and gauge invariance," Physics Letters A, vol. 268, no. 3, pp. 165-173, 2000.

[24] J. T. Lunardi, B. M. Pimentel, J. S. Valverde, and L. A. Manzoni, "Duffin-Kemmer-Petiau theory in the causal approach," International Journal of Modern Physics A, vol. 17, no. 2, pp. 205-227, 2002.

[25] M. de Montigny, F. C. Khanna, A. E. Santana, E. S. Santos, and J. D. M. Vianna, "Galilean covariance and the Duffin-Kemmer-Petiau equation," Journal of Physics A, vol. 33, no. 31, pp. L273-L278, 2000.

[26] Y. Nedjadi and R. C. Barrett, "On the properties of the Duffin-Kemmer-Petiau equation," Journal of Physics G, vol. 19, no. 1, article 006, pp. 87-98, 1993.

[27] Y. Nedjadi, S. Ait-Tahar, and R. C. Barrett, "An extended relativistic quantum oscillator for particles," Journal of Physics A, vol. 31, no. 16, pp. 3867-3874, 1998.

[28] A. Boumali, "On the eigensolutions of the one-dimensional Duffin-Kemmer-Petiau oscillator," Journal of Mathematical Physics, vol. 49, no. 2, Article ID 022302, 2008.

[29] I. Boztosun, M. Karakoc, F. Yasuk, and A. Durmus, "Asymptotic iteration method solutions to the relativistic Duffin-Kemmer-Petiau equation," Journal of Mathematical Physics, vol. 47, no. 6, Article ID 062301, 2006.

[30] B. Boutabia-Chéraitia and T. Boudjedaa, "Solution of DKP equation in Woods-Saxon potential," Physics Letters A, vol. 338, no. 2, pp. 97-107, 2005.

[31] M. Merad, "DKP equation with smooth ptential and position-dependent mass," International Journal of Theoretical Physics, vol. 46, no. 8, pp. 2105-2118, 2007.

[32] Y. Chargui, A. Trabelsi, and L. Chetouani, "Bound-states of the -dimensional DKP equation with a pseudoscalar linear plus Coulomb-like potential," Physics Letters A, vol. 374, no. 29, pp. 2907-2913, 2010.

[33] E. L. Hill, "The theory of vector spherical harmonics," American Journal of Physics, vol. 22, pp. 211-214, 1954.

[34] G. Junker, Supersymmetric Methods in Quantum and Statistical Physics, Springer, 1996.

[35] F. Cooper, A. Khare, and U. Sukhatme, "Supersymmetry and quantum mechanics," Physics Reports A, vol. 251, no. 5-6, pp. 267-385, 1995.

[36] B. K. Bagchi, Supersymmetry in quantum and classical mechanics, Chapman \& Hall, 2000.

[37] E. J. Copeland et al., "Measuring the quantum state of a Bose-Einstein condensate," Physical Review A, vol. 57, pp. 4686-4694, 1998.

[38] Z. Guoa, Y. Piaoa, and Y. Zhang, "Cosmological scaling solutions and multiple exponential potentials," Physics Letters B, vol. 568, no. 1-2, pp. 1-7, 2003.

[39] A. A. Coley, J. Ibáñez, and R. J. van den Hoogen, "Homogeneous scalar field cosmologies with an exponential potential," Journal of Mathematical Physics, vol. 38, no. 10, pp. 5256-5271, 1997.

[40] L. Monchick, "Collision integrals for the exponential repulsive potential," The Physics of Fluids, vol. 2, pp. 695-700, 1959.

[41] J. N. Ginocchio, "Pseudospin as a relativistic symmetry," Physical Review Letters, vol. 78, pp. 436-439, 1997.

[42] R. R. Betts and A. H. Wuosmaa, "Nuclear molecules," Reports on Progress in Physics, vol. 60, no. 8, pp. 819-861, 1997.

[43] C. S. Lam and Y. P. Varshni, "Energies of s eigenstates in a static screened coulomb potential," Physical Review A, vol. 4, no. 5, pp. 1875-1881, 1971.

[44] B. Durand and L. Durand, "Duality for heavy-quark systems," Physical Review D, vol. 23, no. 5, pp. 1092-1102, 1981.

[45] R. L. Hall, "Envelope representations for screened Coulomb potentials," Physical Review A, vol. 32, pp. 14-18, 1985.

[46] A. A. Berezin, "Theory of positron trapping by F- and F/-colour centres in alkali halides," Physica Status Solidi B, vol. 50, no. 1, pp. 71-75, 1972.

[47] N. Hatano and D. R. Nelson, "Localization transitions in Non-Hermitian quantum mechanics," Physical Review Letters, vol. 77, pp. 570-573, 1996.

[48] N. Hatano and D. R. Nelson, "Vortex pinning and non-Hermitian quantum mechanics," Physical Review B, vol. 56, pp. 8651-8673, 1997.

[49] P. M. Morse, "Diatomic molecules according to the wave mechanics. II. Vibrational levels," Physical Review, vol. 34, pp. 57-64, 1929.

[50] S.-H. Dong, R. Lemus, and A. Frank, "Ladder operators for the Morse potential," International Journal of Quantum Chemistry, vol. 86, no. 5, pp. 433-439, 2002.

[51] R. Dutt, K. Chowdhury, and Y. P. Varshni, "An improved calculation for screened Coulomb potentials in Rayleigh-Schrodinger perturbation theory," Journal of Physics A, vol. 18, no. 9, p. 1379, 1985. 
[52] T. Xu, Z.-Q Cao, Y.-C Ou et al., "Critical radius and dipole polarizability for a confined system," Chinese Physics, vol. 15, no. 6, p. 1172, 2006.

[53] T. Tietz, "Negative hydrogen ion," Journal of Chemical Physics, vol. 35, p. 1917, 1961.

[54] K. Szalewicz and H. J. Monkhorst, "On application of 0s orbitals in SCF calculations," The Journal of Chemical Physics, vol. 75, no. 12, pp. 5785-5788, 1981.

[55] G. Malli, "Molecular integrals involving hulthén-type functions $(n=1 S T O)$ in relativistic quantum chemistry," Chemical Physics Letters, vol. 78, no. 3, pp. 578-580, 1981.

[56] J. Lindhard and P. G. Hansen, "Atomic effects in low-energy beta decay: the case of tritium," Physical Review Letters, vol. 57, pp. 965-967, 1986.

[57] I. S. Bitenskya, V. Kh. Ferlegerb, I. A. Wojciechowski et al., “Distortion of $\mathrm{H}_{2}$ potentials by embedding into an electron gas at molecule scattering by a metal surface," Nuclear Instruments and Methods in Physics Research Section B, vol. 125, no. 1-4, pp. 201-206, 1997.

[58] C.-S. Jia, J.-Y. Wang, S. He, and L.-T. Sun, "Shape invariance and the supersymmetry WKB approximation for a diatomic molecule potential," Journal of Physics A, vol. 33, no. 39, pp. 6993-6998, 2000.

[59] P. Pyykko and J. Jokisaari, "Spectral density analysis of nuclear spin-spin coupling: I. A Hulthén potential LCAO model for $J_{X-H}$ in hydrides $\mathrm{XH}_{4}$," Chemical Physics, vol. 10, no. 2-3, pp. 293-301, 1975.

[60] J. A. Olson and D. A. Micha, "Transition operators for atom-atom potentials-the Hilbert-Schmidt expansion," Journal of Chemical Physics, vol. 68, pp. 4352-4356, 1978.

[61] C. Berkdemir, "Pseudospin symmetry in the relativistic Morse potential including the spin-orbit coupling term," Nuclear Physics A, vol. 770, no. 1-2, pp. 32-39, 2006.

[62] O. Bayrak and I. Boztosun, "Application of the asymptotic iteration method to the exponential cosine screened Coulomb potential," International Journal of Quantum Chemistry, vol. 107, no. 5, pp. 10401045, 2007.

[63] C. Berkdemir and H. Jiaguang, "Any 1-state solutions of the Morse potential through the Pekeris approximation and Nikiforov-Uvarov method," Chemical Physics Letters, vol. 409, no. 4-6, pp. $203-$ 207, 2005.

[64] O. Bayrak and I. Boztosun, "The pseudospin symmetric solution of the Morse potential for any $\kappa$ state," Journal of Physics A, vol. 40, no. 36, pp. 11119-11127, 2007.

[65] G. Wei and Sh. Dong, "Pseudospin symmetry in the relativistic Manning-Rosen potential including a Pekeris-type approximation to the pseudo-centrifugal term," Physics Letters B, vol. 686, no. 4-5, pp. 288-292, 2010.

[66] W. Chen and G. Wei, "Spin symmetry in the relativistic modified Rosen-Morse potential with the approximate centrifugal term," Chinese Physics B, vol. 20, Article ID 062101, 2011.

[67] A. Soylu, O. Bayrak, and I. Boztosun, " state solutions of the Dirac equation for the Eckart potential with pseudospin and spin symmetry," Journal of Physics A Mathematical and Theoretical, vol. 41, no. 6, Article ID 065308, 2008.

[68] A. Soylu, O. Bayrak, and I. Boztosun, "An approximate solution of Dirac-Hulthén problem with pseudospin and spin symmetry for any $\kappa$ state," Journal of Mathematical Physics, vol. 48, Article ID 082302, 2009.

[69] H. Egrifes and R. Sever, "Bound-state solutions of the Klein-Gordon equation for the generalized symmetric Hulthén potential," International Journal of Theoretical Physics, vol. 46, no. 4, pp. 935-950, 2007.

[70] A. Okninski, "Supersymmetric content of the Dirac and Duffin-Kemmer-Petiau equations," International Journal of Theoretical Physics, vol. 50, no. 3, pp. 729-736, 2011.

[71] A. Lahiri, P. K. Roy, and B. Bagchi, "Supersymmetry and the three-dimensional isotropic oscillator problem," Journal of Physics, vol. 20, p. 5403, 1987.

[72] A. Lahiri, P. K. Roy, and B. Bagchi, "Supersymmetry in atomic physics and the radial problem," Journal of Physics A, vol. 20, no. 12, pp. 3825-3832, 1987.

[73] V. A. Kostelecky and M. M. Nieto, "Evidence from alkali-metal-atom transition probabilities for a phenomenological atomic supersymmetry," Physical Review A, vol. 32, pp. 1293-1298, 1985.

[74] R. W. Haymaker and A. R. P. Rau, "Supersymmetry in quantum mechanics," American Journal of Physics, vol. 54, p. 928, 1986.

[75] V. A. Kostelecky and M. M. Nieto, "Evidence for a phenomenological supersymmetry in atomic physics," Physical Review Letters, vol. 53, pp. 2285-2288, 1984.

[76] V. A. Kostelecký and M. M. Nieto, "Evidence from alkali-metal-atom transition probabilities for a phenomenological atomic supersymmetry," Physical ReviewA, vol. 32, no. 3, pp. 1293-1298, 1985. 

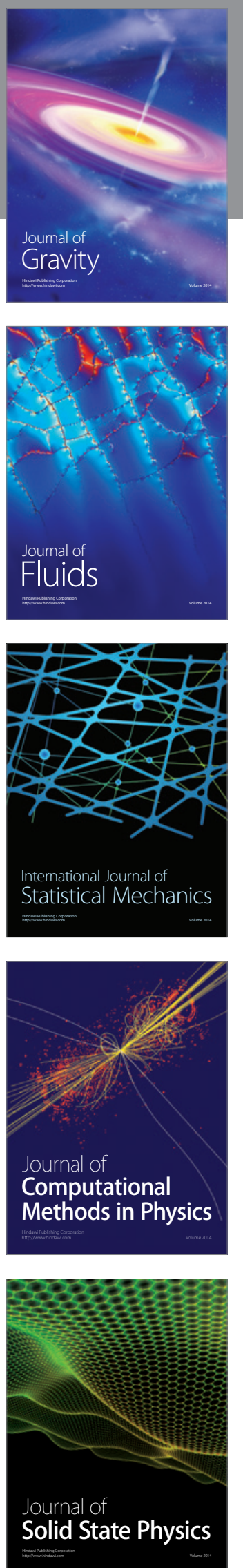
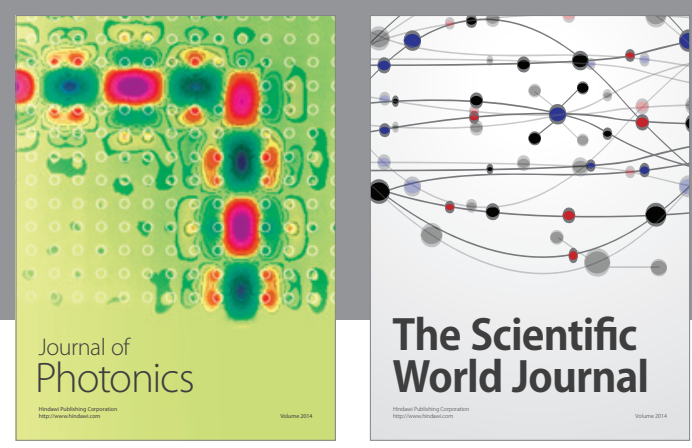

The Scientific World Journal

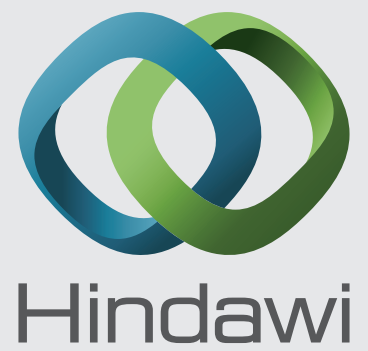

Submit your manuscripts at http://www.hindawi.com
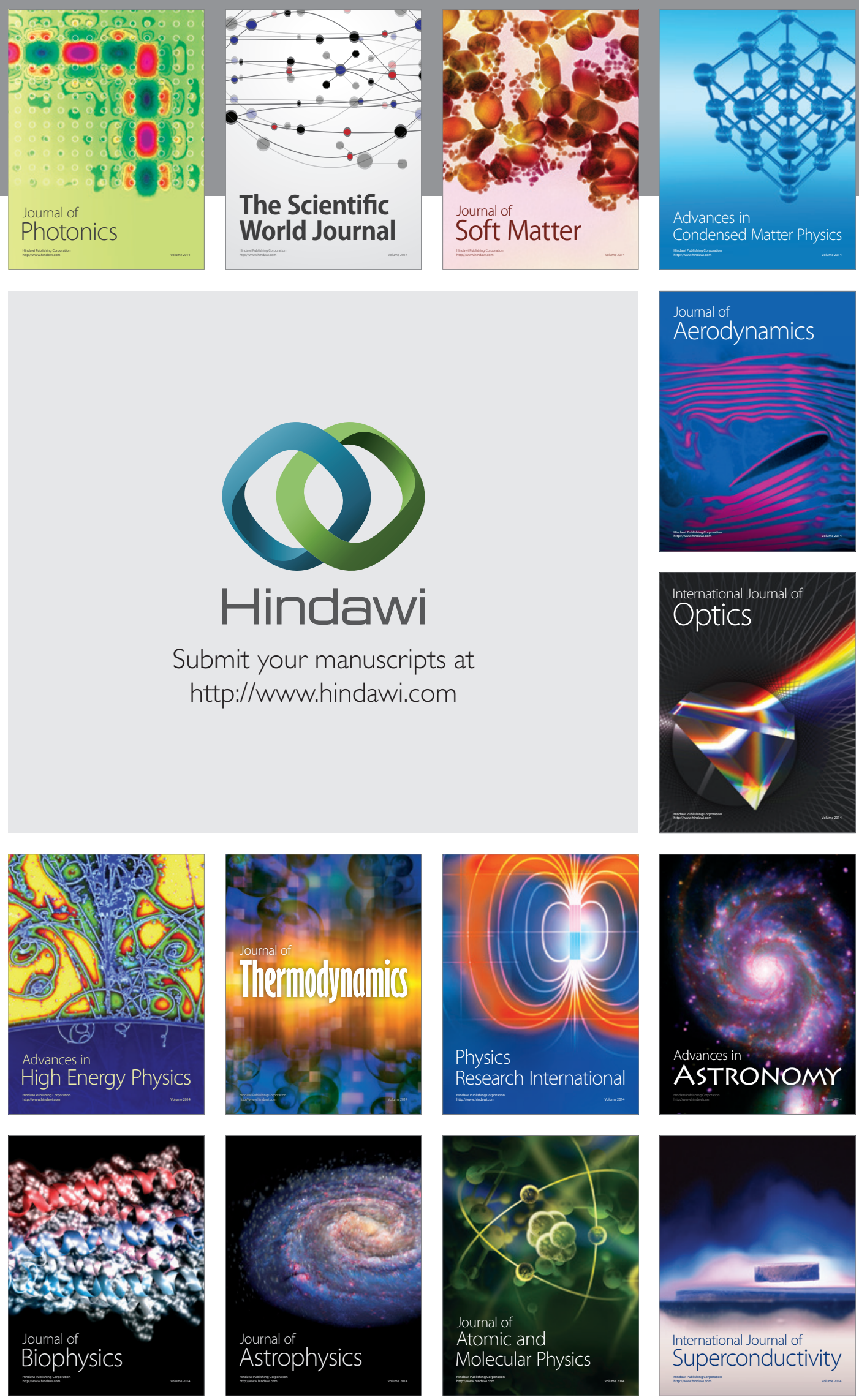
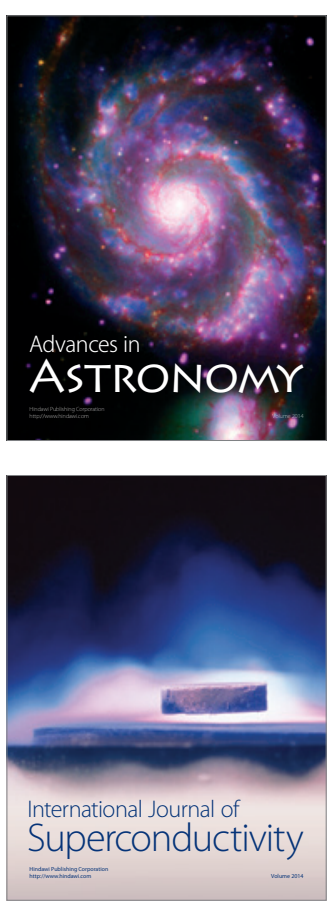